\title{
INFLUENCE OF IMPURITY CHARGE CORRELATION ON FREE-CARRIER ABSORPTION*
}

\author{
W. Szuszkiewicz, P. Sobkowicz, B. Witkowska \\ Institute of Physics, Polish Academy of Sciences \\ Al. Lotników 32/46, 02-668 Warszawa, Poland
}

W. BARDYSZEWSKI

Institute of Theoretical Physics, Warsaw University, Hoża 69, 00-681 Warszawa, Poland

$$
\text { C. JULIEN AND M. BALKANSKI }
$$

Laboratoire de Physique des Solides (unité associé au CNRS 154)

Université Pierre et Marie Curie

4, pl. Jussieu, 75252 Paris, France

\begin{abstract}
Impurity-mediated free-carrier absorption was analyzed theoretically taking into account the possible spatial impurity correlations. The effect of correlations was included via the structure factor $S(q)$ obtained from finite temperature Monte Carlo simulations of ordering in the impurity system. From the comparison of the experimental data with the results of calculations it is demonstrated that the ordering of charged centers exists for $\mathrm{HgSe}: \mathrm{Fe}$ in a wide temperature range.
\end{abstract}

PACS numbers: $71.45 . \mathrm{Gm}, 72.30 .+\mathrm{q}, 75.50 . \mathrm{Pp}$

Impurity mediated scattering may be the dominant mechanism of the free-carrier absorption in a wide doping and temperature range. In calculations of the free-carrier absorption it is usually assumed that the impurities are randomly distributed in space. Recently, a detailed description of this absorption, taking into account possible spatial correlation in the distribution of charges on impurities, as well as collective plasma excitations has been formulated ([1-3] and the references therein). The effect of impurity correlations is included via the structure factor $S(q)$, which is equal to unity in totally uncorrelated systems. The ordering in the positions of the charged impurities drastically reduces the probability of carrier scattering related to small momentum transfers. In this momentum region

*This work is supported in part by the Committee for Scientific Research under grant 204839101 and by the European Economic Community under grant CIPA351OCT920790. 
free-carrier collective excitations (plasmons) are the dominant excitations responsible for the light absorption. The efficiency of plasmon generation in the free-carrier absorption should be therefore strongly reduced for the ordered systems. In particular, it has been suggested that plasmons, being well-defined collective excitations of the electron gas in the range of small wave vectors, can serve as a sensitive tool in studying the space correlation of the Coulomb centers [3].

A well-known example of material in which the spatial impurity charge correlations are present is $\mathrm{HgSe}: \mathrm{Fe}$ (see, e.g. [4-6]). These correlations exist in a mixed valence regime (Fe concentrations greater than about $5 \times 10^{18} \mathrm{~cm}^{-3}$ ) due to the Coulomb repulsion of charges on ionized Fe impurities. The effect of correlations increases with $\mathrm{Fe}$ doping and decreases with increasing temperature. The model proposed in [4-6] enables the theoretical description of correlations at zero temperature limit and cannot be applied to the finite temperature case.

The description of spatial charge correlations used in this paper was based on Monte Carlo simulations, which allow to study the influence of various conditions on the corelation process (for example the change of correlations due to finite temperature). Details of this method may be found in $[7,8]$ and will not be discussed here. We were able to calculate the pair correlation function $g(R)$ and from it the structure factor $S(q)$. An example of results obtained by the method mentioned above is shown in Fig. 1. As one can see, for the iron concentration corresponding
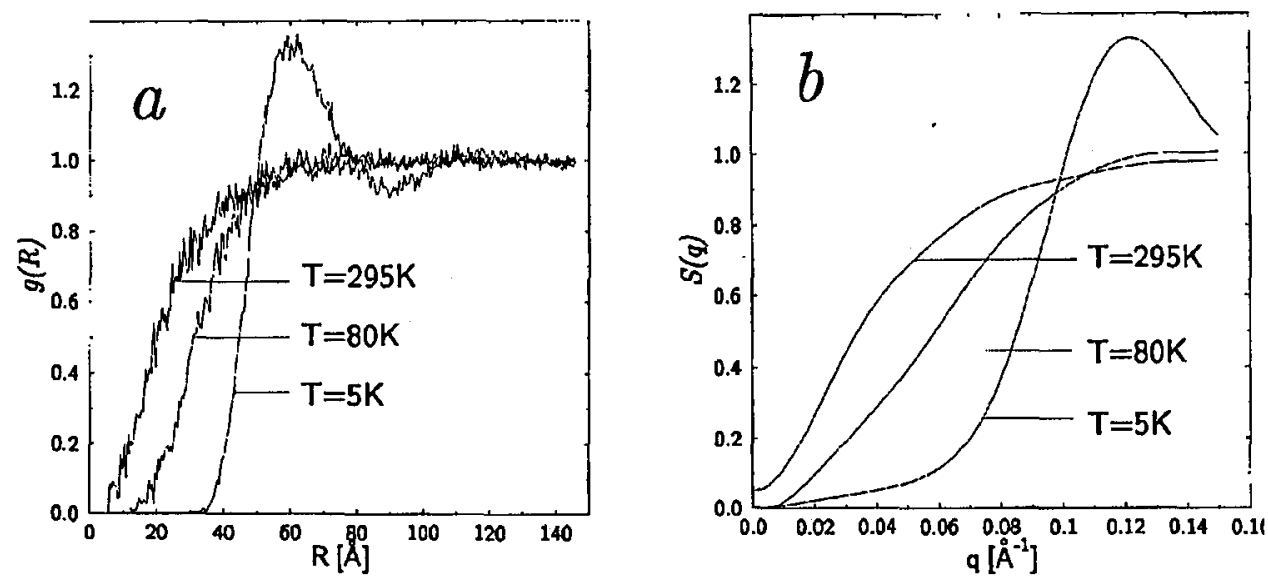

Fig. 1. The temperature dependence of the correlation for HgSe doped with Fe resulting from finite temperature Monte Carlo simulations of ordering in this impurity system (iron concentration $N_{\mathrm{Fe}}=4 \times 10^{19} \mathrm{~cm}^{-3}$, electron concentration $N=5 \times 10^{18} \mathrm{~cm}^{-3}$ ). (a) Pair correlation function $g(R)$, (b) structure factor $S(q)$. The diameter of the Fermi sphere is equal to about $0.1 \AA^{-1}$.

to the mixed valence regime even at room temperature the structure factor $S(q)$ is far from unity in the great part of wave vector range.

Plasmons being the dominant excitations of the electron gas responsible for the free-carrier absorption related to the small momentum transfers should be 
totally suppressed due to negligible value of $S(q)$ in this momentum region. The free-carrier absorption mechanism due to the plasmon excitations should vanish in this case. The impurity mediated contribution to the total free-carrier absorption is analyzed theoretically in Fig. 2a (the neglected phonon contribution is almost energy independent in this frequency range). The frequency dependence of the
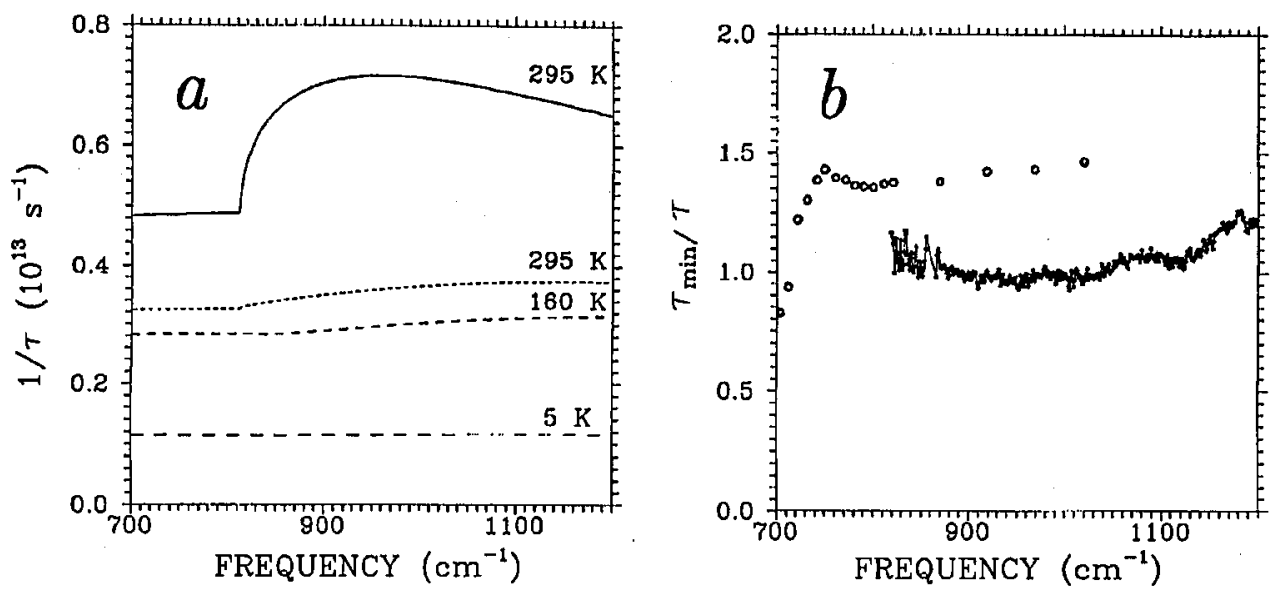

Fig. 2. (a) The results of theoretical calculations of frequency dependent inverse relaxation time for $N_{\mathrm{Fe}}=4 \times 10^{19} \mathrm{~cm}^{-3}$ in the vicinity of the plasma frequency at various temperatures. Solid line - random distribution of ionized centers, dashed lines - spatial charge correlation included. (b) Experimentally determined frequency dependent inverse relaxation time at $T=295 \mathrm{~K}$ for two samples with different $\mathrm{Fe}$ concentration. Open circles: $N_{\mathrm{Fe}}=3 \times 10^{18} \mathrm{~cm}^{-3}$, points: $N_{\mathrm{Fe}}=4 \times 10^{19} \mathrm{~cm}^{-3}$. Both sets of data are subjected to the experimental error close to $15 \%$ due to the finite accuracy of the thin sample thickness measurements (details in text).

inverse relaxation time given by the expression

$$
1 / \tau(\omega)=\frac{\omega^{2} m^{*}}{e^{2} N} \operatorname{Re} \sigma(\omega)=\frac{\omega^{3} m^{*}}{2 \pi e^{2} N} n(\omega) \kappa(\omega),
$$

(where $\sigma$ is the conductivity, $n-$ refraction index, $\kappa$ - extinction coefficient, $N$ - free-carrier concentration) is plotted in this figure in the vicinity of the plasma frequency (formula for $\operatorname{Re} \sigma(\omega)[1,2]$ was applied). $\Lambda$ s one can see, for the random distribution of ionized impurities the sharp increase in the inverse relaxation time value is expected above the plasma frequency. When the possible spatial correlations of charges on $\mathrm{Fe}$ impurity centers are taken into account, even at room temperature this structure is strongly suppressed.

In order to verify these theoretical predictions the free-carrier absorption experiments were performed on HgSe:Fe samples with various Fe concentration. The reflectivity and transmission spectra were measured using the Fourier spectrometer at temperatures from $4.5 \mathrm{~K}$ to $295 \mathrm{~K}$. The values of $1 / \tau$ were determined by the optical constants $n$ and $\kappa$ resulting from the experimental data. For Fe concentrations $N_{\mathrm{Fe}}<5 \times 10^{18} \mathrm{~cm}^{-3}$ due to the lack of correlations the structures in the 
frequency dependence of $1 / \tau$ were well seen at all temperatures. These structures within the experimental error were absent for the sample with $N_{\mathrm{Fe}}=4 \times 10^{19} \mathrm{~cm}^{-3}$. Figure $2 b$ shows the room temperature experimental values of $1 / \tau$ (normalized to its value for the sample with $N_{\mathrm{Fe}}=4 \times 10^{19} \mathrm{~cm}^{-3}$ at the frequency corresponding to the plasma minimum). The plasma frequency for this sample is from 40 to $50 \mathrm{~cm}^{-1}$ smaller than the plasma minimum frequency, equal to about $860 \mathrm{~cm}^{-1}$ at $T=295 \mathrm{~K}$. The small structures seen in Fig. $2 \mathrm{~b}$ are due to the interference phenomena, the slight increase in the value of $1 / \tau$ in the high frequency range results from the contribution due to interband absorption (which was not included in our theoretical calculations). The flat frequency dependence of the experimentally determined $1 / \tau$ for the frequences greater than the plasma frequency for $N_{\mathrm{Fe}}=4 \times 10^{19} \mathrm{~cm}^{-3}$ demonstrates that the correlation of charged centers exists for $\mathrm{HgSe}: \mathrm{Fe}$ in a wide temperature range and confirms the theoretical predictions based on finite temperature Monte Carlo simulations.

\section{References}

[1] S. Goettig, J. Phys. C, Solid State Phys. 17, 4443 (1984).

[2] Bo E. Sernelius, Phys. Rev. B 36, 1080 (1987).

[3] W. Szuszkiewicz, W. Bardyszewski, Qian Dingrong, Zhang Jiaming, C. Julien, M. Balkanski, B. Witkowska, A. Mycielski, in: Proc. 20 ICPS, Thessaloniki 1990, Vol. 3, Eds. E.M. Anastassakis, J.D. Joannopoulos, World Scicntific, Singapore 1990, p. 2255 .

[4] Z. Wilamowski, K. Świątek, T. Dietl, J. Kossut, Solid State Commun. 74, 833 (1990).

[5] Z. Wilamowski, Acta Phys. Pol. A 77, 137 (1990).

[6] J. Kossut, Z. Wilamowski, T. Dietl, K. Świątek, Proc. 20 ICPS, Thessaloniki 1990, Vol. 1, Eds. E.M. Anastassakis, J.D. Joannopoulos, World Scientific, Singapore 1990, p. 613.

[7] P. Sobkowicz, Z. Wilamowski, J. Kossut, Acta Phys. Pol. A 84, (1993).

[8] P. Sobkowicz, Z. Wilamowski, J. Kossut, J. Phys., Condens Matter, in press. 